\title{
Providing 24x7 Access to Engineering Design Lectures Using a Tablet PC and Silicon Chalk
}

\author{
James Blatz ${ }^{1}$ and Ron Britton ${ }^{1 \& 2}$ \\ ${ }^{1}$ Department of Civil Engineering, University of Manitoba, Winnipeg, MB. \\ ${ }^{2}$ Department of Biosystems Engineering, University of Manitoba, Winnipeg, MB. \\ blatzja@cc.umanitoba.ca and ron_britton@umanitoba.ca
}

\begin{abstract}
This paper details the use of Silicon Chalk software in conjunction with a Tablet PC to provide digital lecture files for students in an engineering design course. The advantage of this approach over the traditional overhead or chalkboard is that the notes are digitally recorded by the Tablet PC such that they can be saved following the lecture and distributed to the students for self study. In addition, Silicon Chalk is used to record the audio component of lectures to be distributed to students after the lecture. The result is a classroom environment with increased student participation and interaction.
\end{abstract}

\section{Introduction}

As computing power and capabilities continue to advance at a tremendous rate and the proliferation of software design tools continues, software applications are often used in design offices for even the most routine engineering design activities. Although the fundamentals of the underlying engineering principles still remain the focus of engineering education, exposure to typical software tools in engineering education is paramount to provide graduates with the necessary skills to be quickly productive in the modern workplace. One difficulty with incorporating design software into undergraduate courses is a tendency to spend too much effort on 'how' to use the software as opposed to 'when' and 'why'. Moreover, some have argued that more attention should be given to considerations of when the software should not be used as opposed to when it should be used. In most situations there is limited time to provide detailed instructions on the mechanics of using the software. Demonstrations in the classroom of applications with common software tools are a good approach to teaching the use of design tools. However the ability of the students to adequately document the exercise and at the same time focus on understanding the application being presented is limited. This paper presents the combined use of a new software tool with a Tablet PC that allows the students to focus on understanding concepts presented in the lectures and then use recordings of the lectures to work independently to learn the mechanics of using the software to solve worked problems.

In addition to providing a means to record the use of software in the classroom, Silicon Chalk also provides a change in the classroom dynamic as it relinquishes the requirement for students to take notes during class. This results in increased participation by students in the lecture and better visual feedback for the instructor to gauge student comprehension.

Silicon Chalk is a teaching tool that records the screen and audio streams of a presentation using a standard PC. The software records the lecture in a time indexed format where students can play back the lecture and incorporate their own notes that are time synchronized with the lecture. The power of the software is particularly evident for recording lectures where design software is demonstrated to solve engineering applications. When viewing the recording of the lecture at home the students can independently run the software (with a licensed copy on their local machine) and work through demonstrated examples themselves. This allows time to pause the recording at any point and then mimic the actions in the software itself at their own pace to gain an understanding of how to use the software effectively.

In terms of engineering education the development of concepts are far more important than seeing the final concepts themselves. Silicon Chalk allows students to focus on the development of concepts in the lectures with the knowledge they will be able to independently replay the lectures to learn how to use the software at their own pace. This paper presents a case history of how this process was successfully utilized in a final year Civil Engineering course focused on engineering design concepts. 


\section{The Tablet PC}

The Tablet PC is a lightweight version of a PC that generally is available in two different styles, the slate version and the convertible version. Users interact with a Tablet PC by tapping the screen and writing naturally with a stylus pen

A slate-style tablet appears similar to a large Personal Digital Assistant (PDA). The size varies by manufacturer and model but they are generally 8.5 inches by 12 inches, with a color screen measuring approximately 10 to 12 inches diagonally with control buttons on the edge of the screen. The Tablet PC usually has the ability to connect to a docking station and peripherals including keyboard and mouse. It can also be carried as a mobile device, with a stylus pen for input. The slate design has the advantage of being simple and sleek without hinges or moving parts. It has no keyboard or mouse when the device is mobile, away from the docking station.

The convertible style is essentially a Laptop PC that converts to a Tablet PC. The convertible Tablet PC has a clamshell design similar to the Laptop PC but with a screen that swivels 180 degrees to allow the screen to fold down on top of the keyboard with the screen side facing up. The size of the convertible design is again dependent on manufacturer and model but is similar to the slate. Usually the convertible style is slightly thicker to accommodate the hinged keyboard component. The main advantage of the convertible style is its similarity to the Laptop PC which is familiar to consumers. The convertible style also has the advantage of the attached keyboard, but the hinged clamshell design creates a bulkier device that is susceptible to damage. Design of the hinge and swivel mechanisms varies by manufacturer and model.

In this case example, two Tablet PC's were used for the purpose of comparison. The first was an Acer C110i with a 10.4 inch screen and the second was a Toshiba M200 with a 12.1 inch screen. The details of the comparison are beyond the scope of this paper, however a few broad comments are provided. Both systems provided comparable power and performance. The main difference between the two models was the screen size. When using the units in slate mode during teaching the larger screen size was notably better for development of figures and equations. The corollary to that is the smaller screen size and therefore overall smaller size was beneficial from a mobility standpoint when transiting between lectures.

The Tablet PC was used in the classroom in slate mode when writing out hand written notes in a traditional format and then was used in laptop mode when demonstrating the use of proprietary software for engineering design. Converting the tablet between the two modes takes approximately 15 seconds. Figure 1 shows the Toshiba M200 in both modes. During the lectures, the unit is connected to a multimedia projector so that the screen is displayed at the front of the classroom.

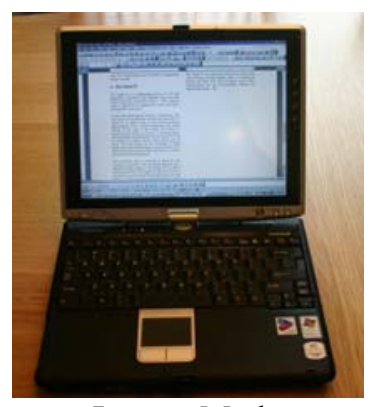

Laptop Mode

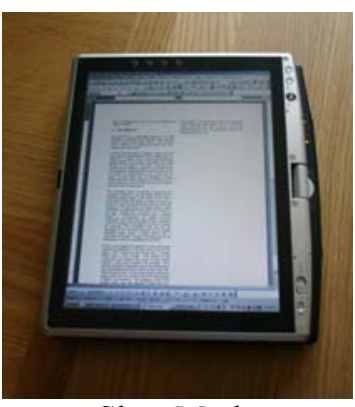

Slate Mode
Figure 1. Tablet PC Modes

\section{Silicon Chalk Software}

Silicon Chalk ${ }^{1}$ (www.siliconchalk.com) is software that transforms the Tablet PC into an invaluable tool in the classroom. It provides interactive tools to encourage participation in the lecture and enhance critical thinking skills. It improves student learning by organizing information presented in the class in an electronic format that can be accessed $24 \times 7$. It ensures that class time is used effectively by keeping students on task. Additionally, it allows remote students to participate in class activities, as if they are in the classroom with network access in the classroom.

In this case example, Silicon Chalk was used as a recording tool to record the Tablet PC screen during all stages of the lectures. The recording basically captures the screen as a movie. The recording includes written notes provided directly on the tablet screen in slate mode and engineering design software demonstrations in laptop mode.

The Silicon Chalk application was setup prior to the term such that the course (Geotechnical Engineering) was identified in the software as an activity. The software was provided to the students in the first tutorial so that they could install it on their home computers and laptops for those who had them. Since the classroom provided wireless network access, students with laptops were able to bring them to the lectures and connect live with the instructor to utilize interactive tools available in the software such as polling, questions, pace, and file sharing. The use of interactive 'live' tools is beyond the focus of this

\footnotetext{
${ }^{1}$ Silicon Chalk Inc., Vancouver, BC, CANADA
} 
discussion but the interested reader is directed to the website for the software (www.siliconchalk.com).

The software has five modes, two of which are pertinent to the discussion in this paper, live mode and recordings. Since the software is set up prior to the term, it takes approximately ten seconds to start the software at the beginning of the lecture to stop the software at the end of the lecture. During the lecture, the software is minimized unless specific tools within the software are being used. Figure 2 shows the interface provided by the software during the lecture. The interface shows tools available along the menu bar and the status of recording in the center of the screen. The software was set-up to record whatever application was in the foreground (active) during the lecture.

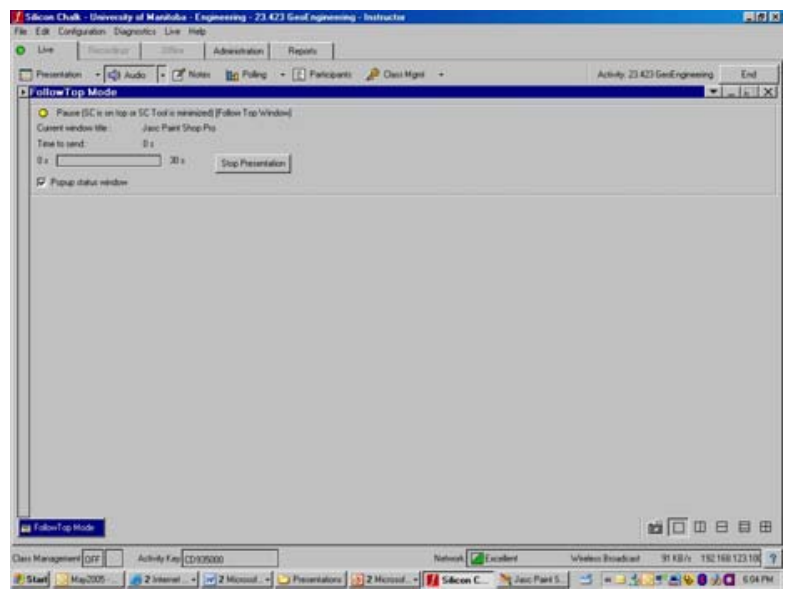

Figure 2. Live mode interface for the instructor.

Once the software is started, students that bring their own laptop to the classroom have the ability to connect to the lecture. The benefit that this provides is that they can type notes into the Silicon Chalk client on their computer. The text notes students take are fully indexed and searchable at a later time. There are also other live features that are available to students when they connect to the instructor including anonymous questions, polling and a pace bar.

During the lecture, the hand written component is provided using Microsoft Journal and is recorded in Silicon Chalk. The hand written component of the lecture is saved at the end of the lecture as a Microsoft Journal file and as an Adobe PDF document. Figure 3 shows an example page from a typical lecture. As noted earlier the particular value of the Silicon Chalk recordings is that the students can replay the lecture to see the development of the lecture with both the audio and the information provided in the lecture. Once the lecture is completed the Silicon Chalk recording file is made available to the students via the course website along with the PDF file.

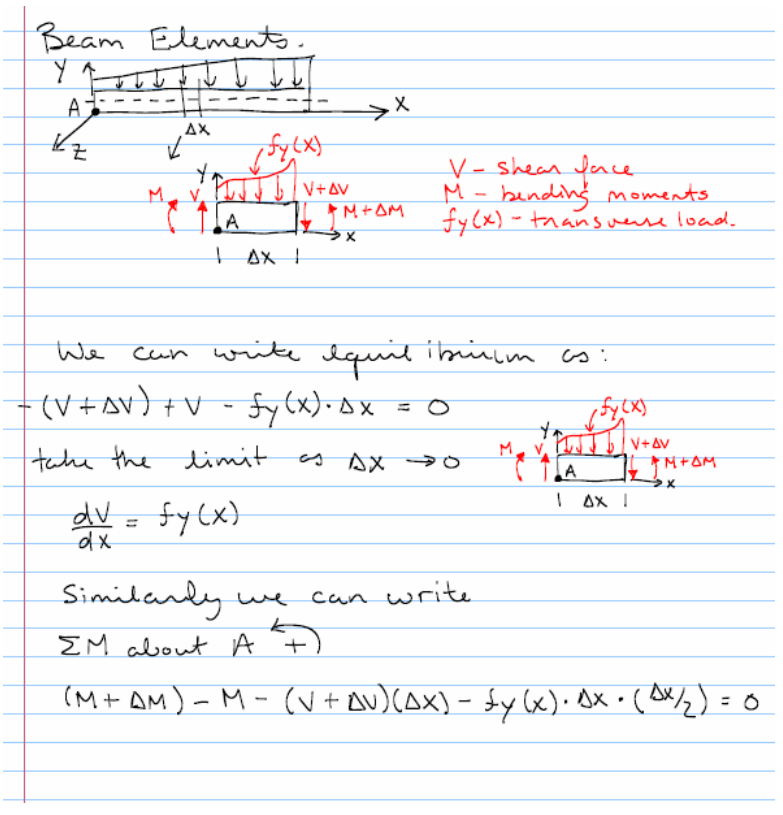

\section{Figure 3. Hand written notes using the Tablet PC and Silicon Chalk.}

The file can be downloaded to a home computer and the student can then view the file in the recording mode. Figure 4 shows an example of what the student would see when replaying a lecture.

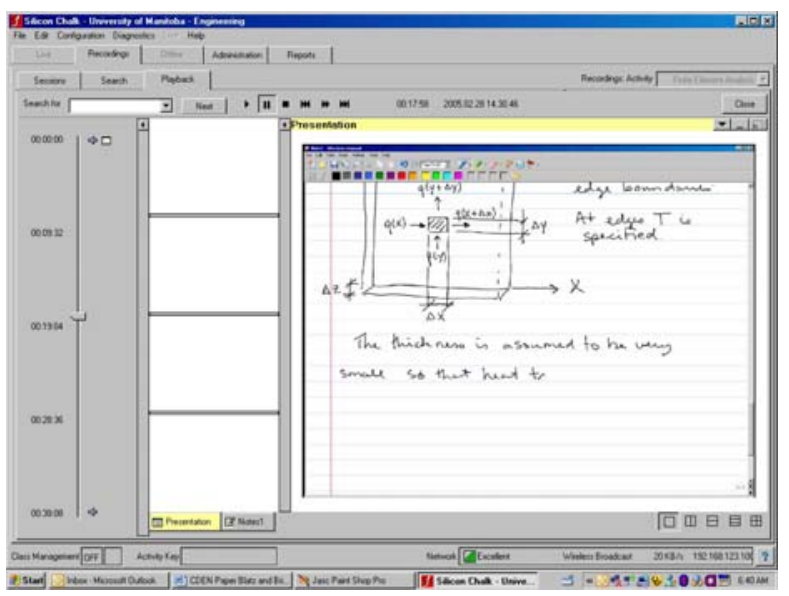

Figure 4. Example lecture play back.

Along the left side of the screen there is a slider bar which shows the total time length of the file corresponding to the lecture. The student can use the slider bar to jump to any time in the lecture. The icons along the slider bar indicate when applications are invoked including the audio recording. By placing the mouse over the icons along the slider bar a description of what application or file was opened at that particular time is displayed. To the right of the slider bar are 
notes windows where the student can enter typed notes. If the student entered typed notes during the lecture, they would appear in these boxes once the lecture file was merged with their local file. To the right of the notes windows is the visual display of what was presented in the lecture, in this case the figure is showing handwritten information. Each lecture is recorded as an individual file and can be made available to the students in a number of ways. A website was established for this course where students could download the written component of the lectures in Adobe PDF format and the Silicon Chalk files which included all of the material presented along with the audio recording. Figure 5 shows the relatively simple webpage developed for this course which was used for distribution of the files.

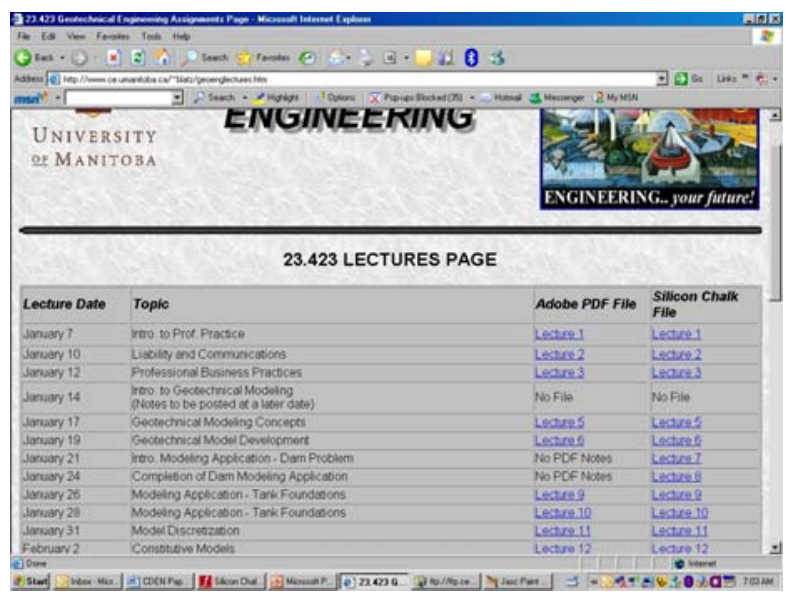

Figure 5. Example of website developed to distribute files for the course.

As noted earlier, the ability to record what is presented on the $\mathrm{PC}$ is of particular value when introducing new software tools for engineering design. In the design course being examined, particular emphasis is placed on the use of limit equilibrium methods for slope stability analysis and finite element analysis for seepage and stress deformation modeling. As such there is considerable time spent in the lectures and laboratory sessions on how to apply these tools to practical applications and how to use them to help train their thinking. When the applications are used during the lectures to solve example problems and demonstrate where and when to apply them, the Silicon Chalk software basically provides a recorded demonstration package for them. Figure 6 shows an example of a slope stability analysis application being discussed in a lecture.

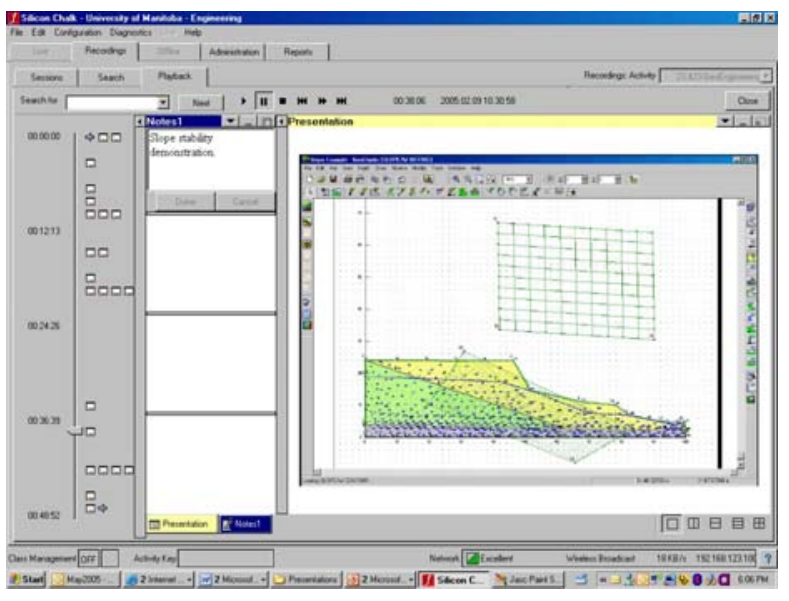

Figure 6. Slope stability application using GeoStudio $^{2}$ (www.geoslope.com)

Along the left side of the screen the slider bar shows considerably more icons indicating that there are a number of applications that are being used during the lecture. Again students can jump to specific points in the lecture corresponding to when specific applications or files were activated by clicking on the corresponding icon. When the student replays the lecture which includes the demonstration of the software for solving the particular problem being examined, they can simultaneously open a copy of the software and work through the problem themselves using the same application. This is particularly beneficial for learning the mechanics of the software as they can listen to the audio instructions and watch what menus are being accessed in the software. At any point the student can stop play back and switch over to the actual software application so that they can replicate what was being done in the lecture to verify their ability to use the software correctly. Students can work at their own respective pace during this activity.

\section{DISCUSSION}

The lecture environment changed dramatically when the Tablet PC and Silicon Chalk were introduced. The use of these tools supported a persistent learning environment where both the 'information as documents' and 'experience as recordings' were provided to the students following the formal lectures.

The benefits to the students included the ability to search the recordings, add their own notes, cross-reference information and most importantly have access to the lectures and lecture information $24 \times 7$.

\footnotetext{
${ }^{2}$ GeoSlope International, Calgary, AB, CANADA
} 
Based on student feedback the most valuable aspect of this approach is that the lectures are available to them at their convenience $24 \times 7$. The fact that students can replay the lectures as a study aid or for catching up on missed classes was particularly valuable.

In the classroom the students were no longer required to focus on writing down all information provided whether it was in hand written form or demonstrations of software tools. This resulted in significantly more interaction amongst the students and professor during the lectures. Some students still felt the need to copy down all information as that best suited their learning style. Students that abandoned writing the notes down were notably more engaged in the lectures. The increased participation tended to actually alter the direction of the lectures to address areas of confusion as identified through the questions. As a result, student participation further improved as the lecture then became an opportunity for students to relay what they did not understand so that the lectures focused in those areas specifically.

From the instructor's standpoint, the improvements are much the same as those the students experience. In addition, the support required to teach the mechanics of software is reduced considerably. Students were better prepared for the hands-on work in the tutorials and were able to better focus their attention to using the software as opposed to learning the software during laboratory periods. The recorded lectures also serve as a permanent record of the material provided during the lectures and as such errors in the notes can be identified and fixed as required. The recordings can be reviewed by the instructor as a self learning tool to identify weaknesses or revisions in the lectures to improve in subsequent years.

As with any new approach that heavily utilizes technology there are some drawbacks worth noting. In order to use the Tablet $\mathrm{PC}$ in conjunction with Silicon Chalk the user must be reasonably comfortable with use of a PC. Once students buy into the use of the software their reliance on the lectures being provided results in an expectation that the electronic files are posted in a timely fashion. Finally there is always a concern that failure of the technology (computer or projector) can occur and contingency plans must be established for that scenario.

\section{Concluding Comments}

The use of a Tablet PC combined with Silicon Chalk to record information provided in lectures along with audio recording of the lecture has been presented.

The benefits of providing recordings of the lectures to the students have been presented. The persistent experience provided by the information as documents and the experience in the lecture as a recording are quite unique. This allows for the students to replay the lectures at home and watch the development of concepts and formulations as opposed to being provided with prepared materials that show only the final product. This feature has proven particularly useful when showing the use of software applications used as design tools in the lectures.

At this point the benefits to the students have been established solely on qualitative observations and informal feedback. By all measures they have indicated acceptance of the approach and have encouraged continued use in the program. Interestingly the common question from fellow academics has been of concern that lecture attendance will diminish since the lecture materials including the recorded audio will be available for download and may be used as a substitute for attendance. On first consideration this would seem obvious, but in fact, the opposite occurred. When the students attend a lecture solely to copy down the notes, then lecture attendance can be replaced with a complete copy of the information provided. When the purpose of attending the lecture is to be engaged in the discussion and to ask questions that direct the lecture to enhance understanding of the concepts being presented, then a recording is not a substitute. In the first scenario it may be difficult for many students to copy feverishly and comprehend to stay engaged in the discussion at the same time. That approach limits the opportunity for students to participate actively in the lecture.

The best measure of the success of this initiative can only be provided by the students who participated in the courses that utilized the approach outlined. The following quote is from a student asked about the value of the approach discussed.

"I find it's great." Second-year student Brent Hay agreed. "When you're sitting there taking notes, I find he's writing them down as quick as I can write them. With this, I can sit there and understand it so much more. It's far more conducive to learning the material. You're paying attention to what's going on and then you can go home, print it out and have all the notes, " Hay said. [1]

Based on this initial experience there is considerable benefit to both the student and instructor in terms of improving the learning environment for engineering design courses.

\section{References}

1. D. Barbour, "The post-chalkboard world", The University of Manitoba Bulletin, Public Affairs, Winnipeg, Manitoba, March 24, 2005, pp. 1-2. 